\title{
Remarkable Classes of Almost 3-Contact Metric Manifolds
}

\section{Giulia Dileo}

Citation: Dileo, G. Remarkable Classes of Almost 3-Contact Metric Manifolds. Axioms 2021, 10, 8. https://doi.org/10.3390/axioms 10010008

Received: 9 December 2020 Accepted: 7 January 2021 Published: 11 January 2021

Publisher's Note: MDPI stays neutral with regard to jurisdictional clai$\mathrm{ms}$ in published maps and institutional affiliations.

Copyright: () 2021 by the author. Licensee MDPI, Basel, Switzerland. This article is an open access article distributed under the terms and conditions of the Creative Commons Attribution (CC BY) license (https:// creativecommons.org/licenses/by/ $4.0 /)$.
Department of Mathematics, University of Bari Aldo Moro, Via E. Orabona 4, 70125 Bari, Italy; giulia.dileo@uniba.it

Abstract: We introduce a new class of almost 3-contact metric manifolds, called 3- $(0, \delta)$-Sasaki manifolds. We show fundamental geometric properties of these manifolds, analyzing analogies and differences with the known classes of 3- $(\alpha, \delta)$-Sasaki $(\alpha \neq 0)$ and 3- $\delta$-cosymplectic manifolds.

Keywords: almost 3-contact metric manifold; 3-Sasaki; 3-cosymplectic; 3- $(\alpha, \delta)$-Sasaki; 3- $\delta$ - cosymplectic; metric connection with skew torsion

MSC: 53C15; 53C25; 53B05

\section{Introduction}

An almost 3-contact metric manifold is a $(4 n+3)$-dimensional differentiable manifold $M$ endowed with three almost contact metric structures $\left(\varphi, \xi_{i}, \eta_{i}, g\right), i=1,2,3$, sharing the same Riemannian metric $g$ and satisfying suitable compatibility conditions, equivalent to the existence of a sphere of almost contact metric structures. In the recent paper [1], new classes of almost 3-contact metric manifolds were introduced and studied. The first remarkable class is given by 3- $(\alpha, \delta)$-Sasaki manifolds defined as almost 3-contact metric manifolds $\left(M, \varphi_{i}, \xi_{i}, \eta_{i}, g\right)$ such that

$$
d \eta_{i}=2 \alpha \Phi_{i}+2(\alpha-\delta) \eta_{j} \wedge \eta_{k}, \quad \alpha \in \mathbb{R}^{*}, \delta \in \mathbb{R},
$$

for every even permutation $(i, j, k)$ of $(1,2,3)$. This is a generalization of 3-Sasaki manifolds, which correspond to the values $\alpha=\delta=1$. A second class introduced in [1] is given by 3- $\delta$-cosymplectic manifolds defined by the conditions

$$
d \eta_{i}=-2 \delta \eta_{j} \wedge \eta_{k}, \quad d \Phi_{i}=0, \quad \delta \in \mathbb{R},
$$

generalizing 3-cosymplectic manifolds which correspond to the value $\delta=0$.

In the present paper we will introduce a third class of almost 3-contact metric manifolds, which is in fact a second (and alternative) generalization of 3-cosymplectic manifolds. We will consider almost 3-contact metric manifolds whose structure tensor fields satisfy

$$
d \eta_{i}=-2 \delta \eta_{j} \wedge \eta_{k}, \quad d \Phi_{i}=-2 \delta\left(\eta_{j} \wedge \Phi_{k}-\eta_{k} \wedge \Phi_{j}\right), \quad \delta \in \mathbb{R}
$$

for every even permutation $(i, j, k)$ of $(1,2,3)$. When $\delta=0$ we recover a 3-cosymplectic manifold. We will call these manifolds $3-(0, \delta)$-Sasaki manifolds. The choice of name is due to the fact that for a 3- $(\alpha, \delta)$-Sasaki manifold, Equation (1) implies

$$
d \Phi_{i}=2(\alpha-\delta)\left(\eta_{j} \wedge \Phi_{k}-\eta_{k} \wedge \Phi_{j}\right)
$$

so that the two equations in (2) formally correspond to (1) and (3) with $\alpha=0$, although in this case the second equation is no more a consequence of the first one. In fact the two conditions in (2) are not completely independent (see Remark 1). Examples of 3- $(0, \delta)$ Sasaki structures can be defined on the semidirect products $\mathrm{SO}(3) \ltimes \mathbb{R}^{4 n}$. The structure on these Lie groups was introduced in [2] as an example of canonical abelian almost 3-contact 
metric structure. It is also shown in [2] that the Lie group $\mathrm{SO}(3) \ltimes \mathbb{R}^{4 n}$ admits co-compact discrete subgroups, so that the corresponding compact quotients admit almost 3-contact metric structures of the same type.

One can show that for all the above three classes of manifolds, $3-(\alpha, \delta)$-Sasaki, 3- $\delta$ cosymplectic, and 3- $(0, \delta)$-Sasaki manifolds, the structure is hypernormal, the characteristic vector fields $\xi_{i}, i=1,2,3$, are Killing and they span an integrable distribution, called vertical, with totally geodesic leaves. Nevertheless, there are remarkable geometric differences between the three classes. In the 3- $(\alpha, \delta)$-Sasaki case the 1 -forms $\eta_{i}$ are all contact forms, i.e., $\eta_{i} \wedge\left(d \eta_{i}\right)^{n} \neq 0$ everywhere on $M$, while for the other two classes, the horizontal distribution defined by $\eta_{i}=0, i=1,2,3$, is integrable. Both 3- $\delta$-cosymplectic manifolds and 3- $(0, \delta)$-Sasaki manifolds are locally isometric to the Riemannian product of a 3-dimensional Lie group, tangent to the vertical distribution, and a $4 n$-dimensional manifold tangent to the horizontal distribution. The Lie group is either isomorphic to $\mathrm{SO}(3)$ or flat depending on wether $\delta \neq 0$ or $\delta=0$. Each horizontal leaf is endowed with a hyper-Kähler structure. The difference between 3- $\delta$-cosymplectic and 3- $(0, \delta)$-Sasaki manifolds lies in the projectability of the structure tensor fields $\varphi_{i}, i=1,2,3$, with respect to the vertical foliation. They are always projectable for 3- $\delta$-cosymplectic manifolds, but not for 3- $(0, \delta)$-Sasaki manifolds with $\delta \neq 0$. In this case one can project a transverse quaternionic structure, as it happens for 3- $(\alpha, \delta)$-Sasaki manifolds. Finally, for the three classes of manifolds, we analyze the existence of a canonical metric connection with totally skew-symmetric torsion.

\section{Almost Contact and Almost 3-Contact Metric Manifolds}

An almost contact manifold is a smooth manifold $M$ of dimension $2 n+1$, endowed with a structure $(\varphi, \xi, \eta)$, where $\varphi$ is a $(1,1)$-tensor field, $\xi$ a vector field, and $\eta$ a 1 -form such that

$$
\varphi^{2}=-I+\eta \otimes \xi, \eta(\xi)=1,
$$

implying that $\varphi \xi=0, \eta \circ \varphi=0$, and $\varphi$ has rank $2 n$. The tangent bundle of $M$ splits as $T M=\mathcal{H} \oplus\langle\xi\rangle$, where $\mathcal{H}$ is the $2 n$-dimensional distribution defined by $\mathcal{H}=\operatorname{Im}(\varphi)=$ $\operatorname{Ker}(\eta)$. The vector field $\xi$ is called the characteristic or Reeb vector field.

On the product manifold $M \times \mathbb{R}$ one can define an almost complex structure $J$ by $J\left(X, f \frac{d}{d t}\right)=\left(\varphi X-f \xi, \eta(X) \frac{d}{d t}\right)$, where $X$ is a vector field tangent to $M, t$ is the coordinate of $\mathbb{R}$ and $f$ is a $\mathcal{C}^{\infty}$ function on $M \times \mathbb{R}$. If $J$ is integrable, the almost contact structure is said to be normal and this is equivalent to the vanishing of the tensor field $N_{\varphi}:=[\varphi, \varphi]+d \eta \otimes \xi$, where $[\varphi, \varphi]$ is the Nijenhuis torsion of $\varphi[3]$. More precisely, for any vector fields $X$ and $Y$, $N_{\varphi}$ is given by

$$
N_{\varphi}(X, Y)=[\varphi X, \varphi Y]+\varphi^{2}[X, Y]-\varphi[\varphi X, Y]-\varphi[X, \varphi Y]+d \eta(X, Y) \xi .
$$

It is known that any almost contact manifold admits a compatible metric, that is a Riemannian metric $g$ such that $g(\varphi X, \varphi Y)=g(X, Y)-\eta(X) \eta(Y)$ for every $X, Y \in \mathfrak{X}(M)$. Then $\eta=g(\cdot, \xi)$ and $\mathcal{H}=\langle\xi\rangle^{\perp}$. The manifold $(M, \varphi, \xi, \eta, g)$ is called an almost contact metric manifold. The associated fundamental 2-form is defined by $\Phi(X, Y)=g(X, \varphi Y)$.

We recall some remarkable classes of almost contact metric manifolds.

- An $\alpha$-contact metric manifold is defined as an almost contact metric manifold such that

$$
d \eta=2 \alpha \Phi, \quad \alpha \in \mathbb{R}^{*},
$$

When $\alpha=1$, it is called a contact metric manifold; the 1 -form $\eta$ is a contact form, that is $\eta \wedge(d \eta)^{n} \neq 0$ everywhere on M. An $\alpha$-Sasaki manifold is a normal $\alpha$-contact metric manifold, and again such a manifold with $\alpha=1$ is called a Sasaki manifold.

- An almost cosymplectic manifold is defined as an almost contact metric manifold such that

$$
d \eta=0, \quad d \Phi=0 ;
$$


if furhermore the structure is normal, $M$ is called a cosymplectic manifold. It is worth remarking that some authors call these manifolds almost coKähler and coKähler, respectively ([4]).

- A quasi-Sasaki manifold is a normal almost contact metric manifold with closed 2-form $\Phi$. This class includes both $\alpha$-Sasaki and cosymplectic manifolds. The Reeb vector field of a quasi-Sasaki manifold is always Killing.

Both $\alpha$-Sasaki manifolds and cosymplectic manifolds can be characterized by means of the Levi-Civita connection $\nabla^{8}$. Indeed, one can show that an almost contact metric manifold $(M, \varphi, \xi, \eta, g)$ is $\alpha$-Sasaki if and only if

$$
\left(\nabla_{X}^{g} \varphi\right) Y=\alpha(g(X, Y) \xi-\eta(X) Y) \quad \forall X, Y \in \mathfrak{X}(M) .
$$

An almost contact metric manifold is cosymplectic if and only if $\nabla^{g} \varphi=0$; further, this is equivalent to requiring the manifold to be locally isometric to the Riemannian product of a real line (tangent to the Reeb vector field) and a Kähler manifold.

For a comprehensive introduction to almost contact metric manifolds we refer to [3]. For Sasaki geometry, we also recommend the monograph [5]; the survey [4] covers fundamental properties and recent results on cosymplectic geometry.

An almost 3-contact manifold is a differentiable manifold $M$ of dimension $4 n+3$ endowed with three almost contact structures $\left(\varphi_{i}, \xi_{i}, \eta_{i}\right), i=1,2,3$, satisfying the following relations,

$$
\begin{gathered}
\varphi_{k}=\varphi_{i} \varphi_{j}-\eta_{j} \otimes \xi_{i}=-\varphi_{j} \varphi_{i}+\eta_{i} \otimes \xi_{j}, \\
\xi_{k}=\varphi_{i} \xi_{j}=-\varphi_{j} \xi_{i}, \quad \eta_{k}=\eta_{i} \circ \varphi_{j}=-\eta_{j} \circ \varphi_{i},
\end{gathered}
$$

for any even permutation $(i, j, k)$ of $(1,2,3)$ ([3]). The tangent bundle of $M$ splits as $T M=\mathcal{H} \oplus \mathcal{V}$, where

$$
\mathcal{H}:=\bigcap_{i=1}^{3} \operatorname{Ker}\left(\eta_{i}\right), \quad \mathcal{V}:=\left\langle\xi_{1}, \xi_{2}, \xi_{3}\right\rangle
$$

In particular, $\mathcal{H}$ has rank $4 n$. We call any vector belonging to the distribution $\mathcal{H}$ horizontal and any vector belonging to the distribution $\mathcal{V}$ vertical. The manifold is said to be hypernormal if each almost contact structure $\left(\phi_{i}, \xi_{i}, \eta_{i}\right)$ is normal. In [6] it was proved that if two of the almost contact structures are normal, then so is the third.

The existence of an almost 3-contact structure is equivalent to the existence of a sphere $\left\{\left(\varphi_{x}, \xi_{x}, \eta_{x}\right)\right\}_{x \in S^{2}}$ of almost contact structures such that

$$
\varphi_{x} \circ \varphi_{y}-\eta_{y} \otimes \xi_{x}=\varphi_{x \times y}-(x \cdot y) I, \quad \varphi_{x} \xi_{y}=\xi_{x \times y}, \quad \eta_{x} \circ \varphi_{y}=\eta_{x \times y},
$$

for every $x, y \in S^{2}$, where $\cdot$ and $\times$ denote the standard inner product and cross product on $\mathbb{R}^{3}$. In fact, if the structure is hypernormal, then every structure in the sphere is normal ([7]).

Any almost 3-contact manifold admits a Riemannian metric $g$ which is compatible with each of the three structures. Then $M$ is said to be an almost 3-contact metric manifold with structure $\left(\varphi_{i}, \xi_{i}, \eta_{i}, g\right), i=1,2,3$. For ease of notation, we will denote an almost 3 -contact metric manifold by $\left(M, \varphi_{i}, \xi_{i}, \eta_{i}, g\right)$, omitting $i=1,2,3$. The subbundles $\mathcal{H}$ and $\mathcal{V}$ are orthogonal with respect to $g$ and the three Reeb vector fields $\xi_{1}, \xi_{2}, \xi_{3}$ are orthonormal. In fact, the structure group of the tangent bundle is reducible to $\operatorname{Sp}(n) \times\{1\}$ [8].

Given an almost 3 -contact metric structure $\left(\varphi_{i}, \xi_{i}, \eta_{i}, g\right)$, an $\mathcal{H}$-homothetic deformation is defined by

$$
\eta_{i}^{\prime}=c \eta_{i}, \quad \xi_{i}^{\prime}=\frac{1}{c} \xi_{i}, \quad \varphi_{i}^{\prime}=\varphi_{i}, \quad g^{\prime}=a g+b \sum_{i=1}^{3} \eta_{i} \otimes \eta_{i},
$$


where $a, b, c$ are real numbers such that $a>0, c^{2}=a+b>0$, ensuring that $\left(\varphi_{i}^{\prime}, \xi_{i}^{\prime}, \eta_{i}^{\prime}, g^{\prime}\right)$ is an almost 3-contact metric structure. In particular, the fundamental 2-forms $\Phi_{i}$ and $\Phi_{i}^{\prime}$ associated to the structures are related by

$$
\Phi_{i}^{\prime}=a \Phi_{i}-b \eta_{j} \wedge \eta_{k}
$$

where $(i, j, k)$ is an even permutation of $(1,2,3)$.

An almost 3-contact metric manifold is called

- $\quad 3$ - $\alpha$-Sasaki, with $\alpha \in \mathbb{R}^{*}$, if $\left(\varphi_{i}, \xi_{i}, \eta_{i}, g\right)$ is $\alpha$-Sasaki for all $i=1,2,3$, i.e. the structure is hypernormal and

$$
d \eta_{i}=2 \alpha \Phi_{i}, \quad i=1,2,3
$$

when $\alpha=1$, it is a 3-Sasaki manifold;

- $\quad 3$-cosymplectic if $\left(\varphi_{i}, \xi_{i}, \eta_{i}, g\right)$ is cosymplectic for all $i=1,2,3$, i.e. the structure is hypernormal and

$$
d \eta_{i}=0, \quad d \Phi_{i}=0, \quad i=1,2,3 ;
$$

- 3-quasi-Sasaki manifold if each structure $\left(\varphi_{i}, \xi_{i}, \eta_{i}, g\right)$ is quasi-Sasaki; this class includes both 3- $\alpha$-Sasaki and 3-cosymplectic manifolds.

These classes were deeply investigated by various authors. See $[5,9,10]$ and references therein for 3-Sasakian geometry, the papers $[7,11,12]$ for 3-cosymplectic manifolds, and $[13,14]$ for 3-quasi-Sasaki manifolds.

In fact, both for 3-Sasaki and 3-cosymplectic manifolds, the hypernormality is consequence of the structure Equations (7) and (8) respectively. This was proved by Kashiwada in [15] for 3-Sasaki manifolds, and in ([16], Theorem 4.13) for 3-cosymplectic manifolds.

In [1] the new classes of 3- $(\alpha, \delta)$-Sasaki manifolds and 3- $\delta$-cosymplectic manifolds were introduced, generalizing the classes of 3- $\alpha$-Sasaki and 3-cosymplectic manifolds, respectively. We will review the definitions and the basic properties of these manifolds in the next section. For both these two classes the hypernormality is a consequence of the defining structure equations for the manifolds, thus generalizing the analogous results for 3-Sasaki and 3-cosymplectic manifolds. This is obtained by using the following Lemma:

Lemma 1 ([1]). Let $\left(M, \varphi_{i}, \xi_{i}, \eta_{i}, g\right)$ be an almost 3-contact metric manifold. Then the following formula holds $\forall X, Y, Z \in \mathfrak{X}(M)$ :

$$
\begin{aligned}
g & \left(N_{\varphi_{i}}(X, Y), Z\right)= \\
= & -d \Phi_{j}\left(X, Y, \varphi_{j} Z\right)+d \Phi_{j}\left(\varphi_{i} X, \varphi_{i} Y, \varphi_{j} Z\right)+d \Phi_{k}\left(X, \varphi_{i} Y, \varphi_{j} Z\right)+d \Phi_{k}\left(\varphi_{i} X, Y, \varphi_{j} Z\right) \\
& -\eta_{i}(X)\left[d \eta_{j}\left(\varphi_{i} Y, \varphi_{j} Z\right)+d \eta_{k}\left(Y, \varphi_{j} Z\right)\right]+\eta_{i}(Y)\left[d \eta_{j}\left(\varphi_{i} X, \varphi_{j} Z\right)+d \eta_{k}\left(X, \varphi_{j} Z\right)\right] \\
& +\eta_{j}(Z)\left[d \eta_{j}(X, Y)-d \eta_{j}\left(\varphi_{i} X, \varphi_{i} Y\right)\right]-\eta_{j}(Z)\left[d \eta_{k}\left(X, \varphi_{i} Y\right)+d \eta_{k}\left(\varphi_{i} X, Y\right)\right] .
\end{aligned}
$$

In the following we will be concerned with various classes of almost 3-contact metric manifolds where the three Reeb vector fields are all Killing. In this case one can show that there exists a function $\delta \in \mathcal{C}^{\infty}(M)$ such that

$$
\eta_{r}\left(\left[\xi_{s}, \xi_{t}\right]\right)=2 \delta \epsilon_{r s t}, \quad r, s, t=1,2,3
$$

where $\epsilon_{r s t}$ is the totally skew-symmetric symbol, or equivalently $d \eta_{r}\left(\xi_{s}, \xi_{t}\right)=-2 \delta \epsilon_{r s t}$. We call $\delta$ a Reeb commutator function, we refer to [1] for more information on this notion.

\section{3- $(\alpha, \delta)$-Sasaki Manifolds and 3- $\delta$-Cosymplectic Manifolds}

This section is a short review of 3- $(\alpha, \delta)$-Sasaki manifolds and 3- $\delta$-cosymplectic manifolds. These were discussed in detail in $[1,17]$. 
Definition 1. An almost 3-contact metric manifold $\left(M, \varphi_{i}, \xi_{i}, \eta_{i}, g\right)$ is called a 3- $(\alpha, \delta)$-Sasaki manifold if it satisfies

$$
d \eta_{i}=2 \alpha \Phi_{i}+2(\alpha-\delta) \eta_{j} \wedge \eta_{k}
$$

for every even permutation $(i, j, k)$ of $(1,2,3)$, where $\alpha \neq 0$ and $\delta$ are real constants.

When $\alpha=\delta=1$, we have a 3-contact metric manifold, and hence a 3-Sasaki manifold by Kashiwada's theorem [15]. In the following, when considering 3- $(\alpha, \delta)$-Sasaki manifolds we will always mean $\alpha \neq 0$. As an immediate consequences of the definition one obtains the following properties:

1. Each $\xi_{i}$ is an infinitesimal automorphism of the distribution $\mathcal{H}$, i. e.

$$
d \eta_{r}\left(X, \xi_{s}\right)=0 \quad X \in \Gamma(\mathcal{H}), r, s=1,2,3 \text {; }
$$

2. The constant $\delta$ is the Reeb commutator function;

3. The differentials $d \Phi_{i}$ are given by

$$
d \Phi_{i}=2(\delta-\alpha)\left(\eta_{k} \wedge \Phi_{j}-\eta_{j} \wedge \Phi_{k}\right)
$$

Applying Lemma 1 one shows the following

Theorem 1 ([1], Theorem 2.2.1). Any 3- $(\alpha, \delta)$-Sasaki manifold is hypernormal.

In particular, a 3- $(\alpha, \delta)$-Sasaki manifold with $\alpha=\delta$ is $3-\alpha$-Sasaki. It can be also shown that the vertical distribution of any 3- $(\alpha, \delta)$-Sasaki manifold is integrable with totally geodesic leaves and each Reeb vector field $\xi_{i}$ is Killing.

We can distinguish three main classes of 3- $(\alpha, \delta)$-Sasaki manifolds. A 3- $(\alpha, \delta)$-Sasaki manifold is called degenerate if $\delta=0$ and non-degenerate otherwise. Quaternionic Heisenberg groups are examples of degenerate 3- $(\alpha, \delta)$-Sasaki manifolds (see ([1], Example 2.3.2)). Considering an $\mathcal{H}$-homothetic deformation of a 3- $(\alpha, \delta)$-Sasaki structure, as in (5), one can verify that the obtained structure $\left(\varphi^{\prime}, \xi^{\prime}, \eta^{\prime}, g^{\prime}\right)$ is a $3-\left(\alpha^{\prime}, \delta^{\prime}\right)$-Sasaki with

$$
\alpha^{\prime}=\alpha \frac{c}{a}, \quad \delta^{\prime}=\frac{\delta}{c} .
$$

In particular, $\mathcal{H}$-homothetic deformations preserve the class of degenerate manifolds. In the nondegenerate case, one sees immediately that $\alpha^{\prime} \delta^{\prime}$ has the same sign as $\alpha \delta$. This justifies the distinction between positive 3- $(\alpha, \delta)$-Sasaki manifolds, with $\alpha \delta>0$, and negative 3- $(\alpha, \delta)$-Sasaki manifolds, with $\alpha \delta<0$. In fact, it can be shown that a 3- $(\alpha, \delta)$-Sasaki manifold is positive if and only if it is $\mathcal{H}$-homothetic to a 3-Sasaki manifold, and negative if and only if it is $\mathcal{H}$-homothetic to a 3- $\left(\alpha^{\prime}, \delta^{\prime}\right)$-Sasaki manifold with $\alpha^{\prime}=-1, \delta^{\prime}=1$.

Examples of negative 3- $(\alpha, \delta)$-Sasaki manifolds can be obtained in the following way. It is known that quaternionic Kähler (not hyper-Kähler) manifolds with negative scalar curvature admit a canonically associated principal SO(3)-bundle $P(M)$ which is endowed with a negative 3-Sasaki structure $[18,19]$. This is a 3-structure $\left(\varphi_{i}, \xi_{i}, \eta_{i}, \tilde{g}\right), i=1,2,3$, where $\left(\varphi_{i}, \xi_{i}, \eta_{i}\right)$ is a normal almost 3-contact structure, and $\tilde{g}$ is a compatible semi-Riemannian metric, with signature $(3,4 n)$, where $4 n$ is the dimension of the base space, and $d \eta_{i}(X, Y)=$ $2 \tilde{g}\left(X, \varphi_{i} Y\right)$. Then, one can define the Riemannian metric

$$
g=-\tilde{g}+2 \sum_{i=1}^{3} \eta_{i} \otimes \eta_{i}
$$

which is compatible with the structure $\left(\varphi_{i}, \xi_{i}, \eta_{i}\right)$, and satisfies $d \eta_{i}=-2 \Phi_{i}-4 \eta_{j} \wedge \eta_{k}$ where $\Phi_{i}(X, Y)=g\left(X, \varphi_{i} Y\right)$ (see also [19]). Therefore $\left(\varphi_{i}, \xi_{i}, \eta_{i}, g\right)$ is a 3 - $(\alpha, \delta)$-Sasaki structure with $\alpha=-1$ and $\delta=1$. 
The following Theorem regarding the transverse geometry with respect to the vertical foliation of a 3- $(\alpha, \delta)$-Sasaki manifold is proved in [17]:

Theorem 2. Any 3- $(\alpha, \delta)$-Sasaki manifold $M$ admits a locally defined Riemannian submersion $\pi: M \rightarrow N$ along its horizontal distribution $\mathcal{H}$ such that $N$ carries a quaternionic Kähler structure given by

$$
\check{\varphi}_{i}=\pi_{*} \circ \varphi_{i} \circ s_{*}, \quad i=1,2,3,
$$

where $s: U \rightarrow M$ is any local smooth section of the Riemannian submersion. The covariant derivatives of the almost complex structures $\breve{\varphi}_{i}$ are given by

$$
\nabla_{X}^{g_{N}} \check{\varphi}_{i}=2 \delta\left(\check{\eta}_{k}(X) \check{\varphi}_{j}-\check{\eta}_{j}(X) \check{\varphi}_{k}\right)
$$

where $\check{\eta}_{i}(X)=\eta_{i}\left(s_{*} X\right)$ os for $i=1,2,3$. The scalar curvature of the base space $N$ is $16 n(n+2) \alpha \delta$.

The Riemannian Ricci tensor of any 3- $(\alpha, \delta)$-Sasaki manifold is computed in [1]:

$$
\operatorname{Ric}^{g}=2 \alpha(2 \delta(n+2)-3 \alpha) g+2(\alpha-\delta)((2 n+3) \alpha-\delta) \sum_{i=1}^{3} \eta_{i} \otimes \eta_{i} .
$$

In particular, a 3- $(\alpha, \delta)$-Sasaki manifold is Riemannian Einstein if and only if $\delta=\alpha$, in which case the structure is 3- $\alpha$-Sasaki, or $\delta=(2 n+3) \alpha$.

Notice that, by Theorem 2, a non-degenerate 3- $(\alpha, \delta)$-Sasaki manifold locally fibers over a quaternionic Kähler space of positive or negative scalar curvature, according to $\alpha \delta>0$ or $\alpha \delta<0$, respectively. In [17] a systematic study of homogeneous non-degenerate 3- $(\alpha, \delta)$-Sasaki manifolds has been carried out, obtaining a complete classification in the positive case, where the base space of the homogeneous fibration turns out to be a symmetric Wolf space. In the case $\alpha \delta<0$, one can provide a general construction of homogeneous 3- $(\alpha, \delta)$-Sasaki manifolds fibering over nonsymmetric Alekseevsky spaces.

We recall now the definition and some basic facts on $3-\delta$-cosymplectic manifolds.

Definition 2. A 3- $\delta$-cosymplectic manifold is an almost 3-contact metric manifold satisfying

$$
d \eta_{i}=-2 \delta \eta_{j} \wedge \eta_{k}, \quad d \Phi_{i}=0
$$

for some $\delta \in \mathbb{R}$ and for every even permutation $(i, j, k)$ of $(1,2,3)$.

When $\delta=0$, the fact that the forms $\eta_{i}$ and $\Phi_{i}$ are all closed implies that the structure is hypernormal ([16], Theorem 4.13). In fact this immediately follows from (9). Therefore, a 3 - $\delta$-cosymplectic manifold with $\delta=0$ is 3-cosymplectic. In particular, it is 3-quasi-Sasaki and the Reeb vector fields are all Killing. The local structure of these manifolds is described by the following:

Proposition 1 ([12]). Any 3-cosymplectic manifold of dimension $4 n+3$ is locally the Riemannian product of a hyper-Kähler manifold of dimension $4 n$ and a 3-dimensional flat abelian Lie group.

As a consequence, since every hyper-Kähler manifold is Ricci flat, even the Riemannian Ricci tensor of any 3-cosymplectic manifold vanishes.

As regards $3-\delta$-cosymplectic manifolds with $\delta \neq 0$, even in this case one can show that the structure is hypernormal, the Reeb vector fields are Killing, and the manifold locally decomposes as a Riemannian product [1]. In particular,

Proposition 2. Any 3- $\delta$-cosymplectic manifold with $\delta \neq 0$ is locally the Riemannian product of a hyper-Kähler manifold and a 3-dimensional Lie group isomorphic to $\mathrm{SO}(3)$, with constant curvature $\delta^{2}$. Consequently, the Riemannian Ricci tensor is $\operatorname{Ric}^{g}=2 \delta^{2} \sum_{i=1}^{3} \eta_{i} \otimes \eta_{i}$. 
In both cases, i.e., $\delta=0$ or $\delta \neq 0$, the hyper-Kähler manifold is tangent to the horizontal distribution, while the 3-dimensional Lie group is tangent to the vertical distribution. In fact, examples of these manifolds can be obtained taking Riemannian products $N \times G$, where $\left(N, J_{i}, h\right), i=1,2,3$, is a hyper-Kähler manifold, and $G$ is a 3-dimensional Lie group, which is either abelian, or isomorphic to $\mathrm{SO}(3)$. If $\xi_{1}, \xi_{2}, \xi_{3}$ are generators of the Lie algebra $\mathfrak{g}$ of $G$, satisfying $\left[\xi_{i}, \xi_{j}\right]=2 \delta \xi_{k}, \delta \in \mathbb{R}$, then one can define in a natural manner an almost 3-contact metric structure $\left(\varphi_{i}, \xi_{i}, \eta_{i}, g\right)$ on the product $N \times G$, setting

$$
\begin{gathered}
\left.\varphi_{i}\right|_{T N}=J_{i}, \quad \varphi_{i} \xi_{i}=0, \quad \varphi_{i} \xi_{j}=\xi_{k}, \quad \varphi_{i} \xi_{k}=-\xi_{j}, \\
\left.\eta_{i}\right|_{T N}=0, \quad \eta_{i}\left(\xi_{i}\right)=1, \quad \eta_{i}\left(\xi_{j}\right)=\eta_{i}\left(\xi_{k}\right)=0,
\end{gathered}
$$

and $g$ the product metric of $h$ and the left invariant Riemannian metric on $G$ with respect to which $\xi_{1}, \xi_{2}, \xi_{3}$ are an orthonormal basis of $\mathfrak{g}$.

For a comparison with the class of 3- $(0, \delta)$-Sasaki manifolds, which will be introduced in the next section, it is worth remarking that for a 3- $\delta$-cosymplectic manifold $\left(M, \varphi_{i}, \xi_{i}, \eta_{i}, g\right)$ the Lie derivatives of the structure tensor fields $\varphi_{i}, i=1,2,3$ with respect to the Reeb vector fields are given by

$$
\mathcal{L}_{\tilde{\zeta}_{i}} \varphi_{i}=0, \quad \mathcal{L}_{\tilde{\zeta}_{i}} \varphi_{j}=2 \delta\left(\eta_{i} \otimes \xi_{j}-\eta_{j} \otimes \xi_{i}\right)=-\mathcal{L}_{\tilde{\zeta}_{j}} \varphi_{i}
$$

for every $i, j=1,2,3$. Indeed, in a $3-\delta$-cosymplectic manifold the Levi-Civita connection satisfies ([1], Proposition 2.1.1):

$$
\begin{gathered}
\nabla_{\xi_{i}}^{g} \varphi_{i}=0, \\
\left(\nabla_{\xi_{i}}^{g} \varphi_{j}\right) X=\delta\left(\eta_{i}(X) \xi_{j}-\eta_{j}(X) \xi_{i}\right)=-\left(\nabla_{\xi_{j}}^{g} \varphi_{i}\right) X, \\
\nabla_{X}^{g} \xi_{i}=\delta\left(\eta_{k}(X) \xi_{j}-\eta_{j}(X) \xi_{k}\right),
\end{gathered}
$$

where $(i, j, k)$ is an even permutation of $(1,2,3)$ and $X \in \mathfrak{X}(M)$. Therefore,

$$
\begin{aligned}
\left(\mathcal{L}_{\xi_{i}} \varphi_{i}\right) X & =\left[\xi_{i}, \varphi_{i} X\right]-\varphi_{i}\left[\xi_{i}, X\right] \\
& =\nabla_{\xi_{i}}^{g}\left(\varphi_{i} X\right)-\nabla_{\varphi_{i} X}^{g} \xi_{i}-\varphi_{i}\left(\nabla_{\xi_{i}}^{g} X\right)+\varphi_{i}\left(\nabla_{X}^{g} \xi_{i}\right) \\
& =\left(\nabla_{\xi_{i}}^{g} \varphi_{i}\right) X-\nabla_{\varphi_{i} X}^{g} \xi_{i}+\varphi_{i}\left(\nabla_{X}^{g} \xi_{i}\right) \\
& =-\delta\left(\eta_{k}\left(\varphi_{i} X\right) \xi_{j}-\eta_{j}\left(\varphi_{i} X\right) \xi_{k}\right)+\delta\left(\eta_{k}(X) \varphi_{i} \xi_{j}-\eta_{j}(X) \varphi_{i} \xi_{k}\right)=0 .
\end{aligned}
$$

In the same way,

$$
\begin{aligned}
\left(\mathcal{L}_{\xi_{i}} \varphi_{j}\right) X & =\left(\nabla_{\xi_{i}}^{g} \varphi_{j}\right) X-\nabla_{\varphi_{j} X}^{g} \xi_{i}+\varphi_{j}\left(\nabla_{X}^{g} \xi_{i}\right) \\
& =\delta\left(\eta_{i}(X) \xi_{j}-\eta_{j}(X) \xi_{i}\right)-\delta \eta_{k}\left(\varphi_{j} X\right) \xi_{j}-\delta \eta_{j}(X) \varphi_{j} \xi_{k} \\
& =2 \delta\left(\eta_{i}(X) \xi_{j}-\eta_{j}(X) \xi_{i}\right)=-\left(\mathcal{L}_{\xi_{j}} \varphi_{i}\right) X .
\end{aligned}
$$

\section{3-(0, $\delta)$-Sasaki Manifolds}

In this section we introduce the class of 3-(0, $\delta)$-Sasaki manifolds.

Definition 3. An almost 3-contact metric manifold $\left(M, \varphi_{i}, \xi_{i}, \eta_{i}, g\right)$ will be called 3-(0, $\left.\delta\right)$-Sasaki manifold if

$$
d \eta_{i}=-2 \delta \eta_{j} \wedge \eta_{k}, \quad d \Phi_{i}=-2 \delta\left(\eta_{j} \wedge \Phi_{k}-\eta_{k} \wedge \Phi_{j}\right)
$$

for every even permutation $(i, j, k)$ of $(1,2,3)$, and for some real constant $\delta \in \mathbb{R}$.

In particular, the structure is not 3-quasi-Sasaki when $\delta \neq 0$, and we have the following basic properties for a 3- $(0, \delta)$-Sasaki manifold:

1. The horizontal distribution $\mathcal{H}$ is integrable; 
2. Each $\xi_{i}$ is an infinitesimal automorphism of the distribution $\mathcal{H}$, i. e.

$$
d \eta_{r}\left(X, \xi_{s}\right)=0 \quad X \in \Gamma(\mathcal{H}), r, s=1,2,3 ;
$$

3. The constant $\delta$ is the Reeb commutator function.

Remark 1. In case $\delta \neq 0$, the two equations in (12) are not completely independent. Indeed, if one assumes $d \Phi_{i}=-2 \gamma\left(\eta_{j} \wedge \Phi_{k}-\eta_{k} \wedge \Phi_{j}\right), \gamma \in \mathbb{R}^{*}$, differentiating this equation, and combining with $d \eta_{i}=-2 \delta \eta_{j} \wedge \eta_{k}$, a straightforward computation gives $\gamma=\delta$. Thus, there is no freedom for the choice of constant in the second equation.

If $\left(\varphi_{i}, \xi_{i}, \eta_{i}, g\right)$ is a 3-(0, $\left.\delta\right)$-Sasaki structure, applying an $\mathcal{H}$-homothetic deformation as in (5), an easy computation using (6) shows that the new structure $\left(\varphi_{i}^{\prime}, \eta_{i}^{\prime}, \xi_{i}^{\prime}, g^{\prime}\right)$ is again 3- $\left(0, \delta^{\prime}\right)-$ Sasaki, with $\delta^{\prime}=\frac{\delta}{c}$.

Example 1. Consider the abelian Lie algebra $\mathbb{R}^{4 n}$ spanned by vectors $v_{r}, v_{n+r}, v_{2 n+r}, v_{3 n+r}$, $r=1, \ldots, n$, and endowed with the hypercomplex structure $\left\{J_{1}, J_{2}, J_{3}\right\}$ defined by

$$
J_{i}\left(v_{r}\right)=v_{i n+r}, \quad J_{i}\left(v_{i n+r}\right)=-v_{r}, \quad J_{i}\left(v_{j n+r}\right)=v_{k n+r}, \quad J_{i}\left(v_{k n+r}\right)=-v_{j n+r},
$$

for every even permutation $(i, j, k)$ of $(1,2,3)$. Let us consider also the Lie algebra $\mathfrak{s o}(3)$ spanned by $\xi_{1}, \xi_{2}, \xi_{3}$ with Lie brackets given by $\left[\xi_{i}, \xi_{j}\right]=2 \delta \xi_{k}, \delta \neq 0$. Let $\rho$ be the representation of $\mathfrak{s o}(3)$ on $\mathbb{R}^{4 n}$ given by

$$
\rho: \mathfrak{s o}(3) \rightarrow \mathfrak{g l}(4 n, \mathbb{R}), \quad \rho\left(\xi_{i}\right)=\delta J_{i}, \quad i=1,2,3 .
$$

On the Lie algebra $\mathfrak{g}=\mathfrak{s o}(3) \ltimes_{\rho} \mathbb{R}^{4 n}$ on can define in a natural way an almost 3-contact metric structure $\left(\varphi_{i}, \xi_{i}, \eta_{i}, g\right)$, with

$$
\begin{gathered}
\left.\varphi_{i}\right|_{\mathbb{R}^{4 n}}=J_{i}, \quad \varphi\left(\xi_{i}\right)=0, \quad \varphi_{i}\left(\xi_{j}\right)=\xi_{k}=-\varphi_{j}\left(\xi_{k}\right), \\
\left.\eta_{i}\right|_{\mathbb{R}^{4 n}}=0, \quad \eta_{i}\left(\xi_{i}\right)=1, \quad \eta_{i}\left(\xi_{j}\right)=\eta_{i}\left(\xi_{k}\right)=0,
\end{gathered}
$$

and where $g$ is the inner product such that the vectors $\xi_{i}, v_{l}, i=1,2,3, l=1, \ldots, 4 n$ are orthonormal. In particular, the non zero brackets on $\mathfrak{g}$ are given by

$$
\left[\xi_{i}, \xi_{j}\right]=2 \delta \xi_{k}, \quad\left[\xi_{i}, X\right]=\delta \varphi_{i}(X), X \in \mathbb{R}^{4 n} .
$$

The representation $\rho: \mathfrak{s o}(3) \rightarrow \mathfrak{g l}(4 n, \mathbb{R})$ can be integrated to a representation $\tilde{\rho}: \mathrm{SO}(3) \rightarrow$ $\mathrm{GL}(4 n, \mathbb{R})$. Therefore, identifying $\mathbb{R}^{4 n}$ with $\mathbb{H}^{n}$ in a natural way, the simply connected Lie group $G=\mathrm{SO}(3) \ltimes_{\tilde{\rho}} \mathbb{H}^{n}$, with Lie algebra $\mathfrak{g}$, admits a left invariant almost 3-contact metric structure $\left(\varphi_{i}, \xi_{i}, \eta_{i}, g\right)$. One can easily verify that this structure satisfies (12).

Remark 2. For more details on the above example we refer to [2], where $\mathfrak{g}$ is described as a remarkable example of a Lie algebra endowed with an abelian almost 3-contact metric structure. In fact, the structure defined on $\mathfrak{g}$ belongs to the class of canonical abelian structures, so that the Lie group $G$ admits a unique metric connection with totally skew symmetric torsion $\nabla$ such that

$$
\nabla_{X} \varphi_{i}=2 \delta\left(\eta_{k}(X) \varphi_{j}-\eta_{j}(X) \varphi_{k}\right)
$$

for every vector field $X$ and for every even permutation $(i, j, k)$ of $(1,2,3)$. The torsion of the canonical connection $\nabla$ is $T=2 \delta \eta_{1} \wedge \eta_{2} \wedge \eta_{3}$, which satisfies $\nabla T=0$.

It is also shown in [2] that the Lie group $G$ admits co-compact discrete subgroups, so that the corresponding compact quotients admit almost 3-contact metric structures of the same type.

Proposition 3. Let $\left(M, \varphi_{i}, \xi_{i}, \eta_{i}, g\right)$ be a 3-(0, $)$-Sasaki manifold. Then the structure is hypernormal. 
Proof. In order to compute the tensor fields $N_{\varphi_{i}}$, we apply Lemma 1 . We always denote by $X, Y, Z$ horizontal vector fields and by $(i, j, k)$ an even permutation of $(1,2,3)$.

Being $d \Phi_{i}(X, Y, Z)=0$, then $N_{\varphi_{i}}(X, Y, Z)=0$ for every $i=1,2,3$. Furthermore, since the horizontal distribution is integrable, by the definition of the tensor field $N_{\varphi_{i}}$ (see (4)), one has $N_{\varphi_{i}}\left(X, Y, \xi_{r}\right)=0$ for all $r=1,2,3$. Notice that, since

$$
\left.\left.\left.\xi_{i}\right\lrcorner \Phi_{i}=0, \quad \xi_{j}\right\lrcorner \Phi_{i}=-\eta_{k}, \quad \xi_{k}\right\lrcorner \Phi_{i}=\eta_{j},
$$

from the second equation in (12), we have,

$$
\left.\left.\left.\xi_{i}\right\lrcorner d \Phi_{i}=0, \quad \xi_{j}\right\lrcorner d \Phi_{i}=-2 \delta\left(\Phi_{k}+\eta_{i j}\right), \quad \xi_{k}\right\lrcorner d \Phi_{i}=2 \delta\left(\Phi_{j}+\eta_{k i}\right) .
$$

Therefore, form Lemma 1, applying (12) and (13), we have

$$
\begin{aligned}
N_{\varphi_{i}}\left(X, \xi_{i}, Z\right) & =-d \Phi_{j}\left(X, \xi_{i}, \varphi_{j} Z\right)+d \Phi_{k}\left(\varphi_{i} X, \xi_{i}, \varphi_{j} Z\right)+d \eta_{j}\left(\varphi_{i} X, \varphi_{j} Z\right)+d \eta_{k}\left(X, \varphi_{j} Z\right) \\
& =-2 \delta \Phi_{k}\left(\varphi_{j} Z, X\right)-2 \delta \Phi_{j}\left(\varphi_{j} Z, \varphi_{i} X\right) \\
& =2 \delta \Phi_{j}\left(\varphi_{i} X, \varphi_{j} Z\right)+2 \delta \Phi_{k}\left(X, \varphi_{j} Z\right)=-2 \delta g\left(\varphi_{i} X, Z\right)-2 \delta g\left(X, \varphi_{i} Z\right)=0, \\
N_{\varphi_{i}}\left(X, \xi_{j}, Z\right) & =d \Phi_{j}\left(\varphi_{i} X, \xi_{k}, \varphi_{j} Z\right)+d \Phi_{k}\left(\varphi_{i} X, \xi_{j}, \varphi_{j} Z\right) \\
& =-2 \delta \Phi_{i}\left(\varphi_{j} Z, \varphi_{i} X\right)+2 \delta \Phi_{i}\left(\varphi_{j} Z, \varphi_{i} X\right)=0, \\
N_{\varphi_{i}}\left(X, \xi_{k}, Z\right) & =-d \Phi_{j}\left(X, \xi_{k}, \varphi_{j} Z\right)-d \Phi_{k}\left(X, \xi_{j}, \varphi_{j} Z\right) \\
& =2 \delta \Phi_{i}\left(\varphi_{j} Z, X\right)-2 \delta \Phi_{i}\left(\varphi_{j} Z, X\right)=0 .
\end{aligned}
$$

Equations (13) implies $d \Phi_{r}\left(X, \xi_{s}, \xi_{t}\right)=0$ for every $r, s, t=1,2,3$ and $X \in \Gamma(\mathcal{H})$. Taking also into account that $d \eta_{r}\left(X, \xi_{s}\right)=0$, we deduce from (9) that

$$
N_{\varphi_{r}}\left(X, \xi_{s}, \xi_{t}\right)=N_{\varphi_{r}}\left(\xi_{s}, \xi_{t}, X\right)=0
$$

Finally, (9) implies together with $d \eta_{r}\left(\xi_{s}, \xi_{t}\right)=-2 \delta \epsilon_{r s t}$ that

$$
N_{\varphi_{i}}\left(\xi_{i}, \xi_{j}, \xi_{k}\right)=N_{\varphi_{i}}\left(\xi_{i}, \xi_{k}, \xi_{j}\right)=N_{\varphi_{i}}\left(\xi_{j}, \xi_{k}, \xi_{i}\right)=0,
$$

completing the proof that $M$ is hypernormal.

Proposition 4. Let $\left(M, \varphi_{i}, \xi_{i}, \eta_{i}, g\right)$ be a 3-(0, $\left.\delta\right)$-Sasaki manifold. Then the Levi-Civita connection satisfies for all $X, Y \in \mathfrak{X}(M)$ and any cyclic permutation $(i, j, k)$ of $(1,2,3)$ :

$$
\begin{aligned}
\left(\nabla_{X}^{g} \varphi_{i}\right) Y= & 2 \delta\left[\eta_{k}(X) \varphi_{j} Y-\eta_{j}(X) \varphi_{k} Y\right] \\
& -\delta\left[\eta_{j}(X) \eta_{j}(Y)+\eta_{k}(X) \eta_{k}(Y)\right] \xi_{i}+\delta \eta_{i}(Y)\left[\eta_{j}(X) \xi_{j}+\eta_{k}(X) \xi_{k}\right]
\end{aligned}
$$

and

$$
\begin{gathered}
\nabla_{X}^{g} \xi_{i}=\delta\left(\eta_{k}(X) \xi_{j}-\eta_{j}(X) \xi_{k}\right), \\
\nabla_{\xi_{i}}^{g} \xi_{i}=0, \quad \nabla_{\xi_{i}}^{g} \xi_{j}=-\nabla_{\xi_{j}}^{g} \xi_{i}=\delta \xi_{k} .
\end{gathered}
$$

In particular, each $\xi_{i}$ is a Killing vector field.

Proof. Since the structure is hypernormal, by ([3], Lemma 6.1), the Levi-Civita connection satisfies

$$
\begin{aligned}
2 g\left(\left(\nabla_{X}^{g} \varphi_{i}\right) Y, Z\right)= & d \Phi_{i}\left(X, \varphi_{i} Y, \varphi_{i} Z\right)-d \Phi_{i}(X, Y, Z) \\
& +d \eta_{i}\left(\varphi_{i} Y, X\right) \eta_{i}(Z)-d \eta_{i}\left(\varphi_{i} Z, X\right) \eta_{i}(Y)
\end{aligned}
$$

Further, an easy computation (see [1]) shows that for every cyclic permutation $(i, j, k)$ of $(1,2,3)$, 


$$
\begin{aligned}
\Phi_{j}\left(\varphi_{i} X, \varphi_{i} Y\right) & =-\Phi_{j}(X, Y)-\left(\eta_{k} \wedge \eta_{i}\right)(X, Y), \\
\Phi_{k}\left(\varphi_{i} X, \varphi_{i} Y\right) & =-\Phi_{k}(X, Y)-\left(\eta_{i} \wedge \eta_{j}\right)(X, Y), \\
\Phi_{j}\left(\varphi_{i} X, Y\right) & =-\Phi_{k}(X, Y)-\eta_{i}(X) \eta_{j}(Y), \\
\Phi_{k}\left(\varphi_{i} X, Y\right) & =\Phi_{j}(X, Y)-\eta_{i}(X) \eta_{k}(Y) .
\end{aligned}
$$

Then, using the second equation in (12) and the above equations, we have

$$
\begin{aligned}
d \Phi_{i} & \left(X, \varphi_{i} Y, \varphi_{i} Z\right)= \\
= & -2 \delta\left[\eta_{j}(X) \Phi_{k}\left(\varphi_{i} Y, \varphi_{i} Z\right)+\eta_{j}\left(\varphi_{i} Y\right) \Phi_{k}\left(\varphi_{i} Z, X\right)+\eta_{j}\left(\varphi_{i} Z\right) \Phi_{k}\left(X, \varphi_{i} Y\right)\right. \\
& \left.-\eta_{k}(X) \Phi_{j}\left(\varphi_{i} Y, \varphi_{i} Z\right)-\eta_{k}\left(\varphi_{i} Y\right) \Phi_{j}\left(\varphi_{i} Z, X\right)-\eta_{k}\left(\varphi_{i} Z\right) \Phi_{j}\left(X, \varphi_{i} Y\right)\right] \\
= & -2 \delta\left[-\eta_{j}(X) \Phi_{k}(Y, Z)-\eta_{j}(X)\left(\eta_{i} \wedge \eta_{j}\right)(Y, Z)\right. \\
& -\eta_{k}(Y) \Phi_{j}(Z, X)+\eta_{k}(Y) \eta_{i}(Z) \eta_{k}(X)+\eta_{k}(Z) \Phi_{j}(Y, X)-\eta_{k}(Z) \eta_{i}(Y) \eta_{k}(X) \\
& +\eta_{k}(X) \Phi_{j}(Y, Z)+\eta_{k}(X)\left(\eta_{k} \wedge \eta_{i}\right)(Y, Z) \\
& \left.+\eta_{j}(Y) \Phi_{k}(Z, X)+\eta_{j}(Y) \eta_{i}(Z) \eta_{j}(X)-\eta_{j}(Z) \Phi_{k}(Y, X)-\eta_{j}(Z) \eta_{i}(Y) \eta_{j}(X)\right] \\
= & d \Phi_{i}(X, Y, Z)+4 \delta\left[\eta_{j}(X) \Phi_{k}(Y, Z)-\eta_{k}(X) \Phi_{j}(Y, Z)\right] \\
& +4 \delta \eta_{j}(X)\left[\eta_{i}(Y) \eta_{j}(Z)-\eta_{j}(Y) \eta_{i}(Z)\right] \\
& +4 \delta \eta_{k}(X)\left[\eta_{i}(Y) \eta_{k}(Z)-\eta_{k}(Y) \eta_{i}(Z)\right] .
\end{aligned}
$$

On the other hand, again using the first equation in (12), we obtain

$$
\begin{aligned}
& d \eta_{i}\left(\varphi_{i} Y, X\right) \eta_{i}(Z)-d \eta_{i}\left(\varphi_{i} Z, X\right) \eta_{i}(Y)= \\
& =-2 \delta\left(\eta_{j} \wedge \eta_{k}\right)\left(\varphi_{i} Y, X\right) \eta_{i}(Z)+2 \delta\left(\eta_{j} \wedge \eta_{k}\right)\left(\varphi_{i} Z, X\right) \eta_{i}(Y) \\
& =-2 \delta \eta_{i}(Z)\left[-\eta_{k}(Y) \eta_{k}(X)-\eta_{j}(X) \eta_{j}(Y)\right]+2 \delta \eta_{i}(Y)\left[-\eta_{k}(Z) \eta_{k}(X)-\eta_{j}(X) \eta_{j}(Z)\right] .
\end{aligned}
$$

Inserting the above computations in (17), we conclude that

$$
\begin{aligned}
g\left(\left(\nabla_{X}^{g} \varphi_{i}\right) Y, Z\right) & =2 \delta\left[\eta_{k}(X) g\left(\varphi_{j} Y, Z\right)-\eta_{j}(X) g\left(\varphi_{k} Y, Z\right)\right] \\
& -\delta \eta_{i}(Z)\left[\eta_{k}(Y) \eta_{k}(X)+\eta_{j}(X) \eta_{j}(Y)\right]+\delta \eta_{i}(Y)\left[\eta_{k}(Z) \eta_{k}(X)+\eta_{j}(X) \eta_{j}(Z)\right]
\end{aligned}
$$

which implies (14). As regards the proof (15), applying (14) for $Y=\xi_{i}$, we get

$$
\left(\nabla_{X}^{g} \varphi_{i}\right) \xi_{i}=-\delta\left(\eta_{j}(X) \xi_{j}+\eta_{k}(X) \xi_{k}\right)
$$

Applying $\varphi_{i}$ on both hand-sides, we obtain (15). Equations (16) are immediate consequences of (15). Furthermore, we also get

$$
g\left(\nabla_{X}^{g} \xi_{i}, Y\right)=-\delta\left(\eta_{j} \wedge \eta_{k}\right)(X, Y)
$$

for every $X, Y \in \mathfrak{X}(M)$. Since $\nabla^{g} \xi_{i}$ is skew-symmetric, $\xi_{i}$ is Killing.

Corollary 1. Let $\left(M, \varphi_{i}, \xi_{i}, \eta_{i}, g\right)$ be a 3-(0, $\left.\delta\right)$-Sasaki manifold. Then for every even permutation $(i, j, k)$ of $(1,2,3)$ we have

$$
\mathcal{L}_{\xi_{i}} \varphi_{i}=0, \quad \mathcal{L}_{\xi_{i}} \varphi_{j}=-\mathcal{L}_{\xi_{j}} \varphi_{i}=2 \delta \varphi_{k} .
$$

Proof. For the first Lie derivative, notice that by (14) we have $\nabla_{\xi_{i}}^{g} \varphi_{i}=0$. Then, applying also (15), for every vector field $X$ we have

$$
\begin{aligned}
\left(\mathcal{L}_{\tilde{\xi}_{i}} \varphi_{i}\right) X & =\left(\nabla_{\xi_{i}}^{g} \varphi_{i}\right) X-\nabla_{\varphi_{i} X}^{g} \xi_{i}+\varphi_{i}\left(\nabla_{X}^{g} \xi_{i}\right) \\
& =-\delta\left(\eta_{k}\left(\varphi_{i} X\right) \xi_{j}-\eta_{j}\left(\varphi_{i} X\right) \xi_{k}\right)+\delta\left(\eta_{k}(X) \varphi_{i} \xi_{j}-\eta_{j}(X) \varphi_{i} \xi_{k}\right)=0 .
\end{aligned}
$$


Now, using (14) for the covariant derivative $\nabla^{g} \varphi_{j}$, for every vector field $Y$, we have

$$
\left(\nabla_{\xi_{i}}^{g} \varphi_{j}\right) Y=2 \delta \varphi_{k} Y-\delta\left(\eta_{i}(Y) \xi_{j}-\eta_{j}(Y) \xi_{i}\right) .
$$

Therefore, applying also (15), we get

$$
\begin{aligned}
\left(\mathcal{L}_{\xi_{i}} \varphi_{j}\right) X & =\left(\nabla_{\xi_{i}}^{g} \varphi_{j}\right) X-\nabla_{\varphi_{j} X}^{g} \xi_{i}+\varphi_{j}\left(\nabla_{X}^{g} \xi_{i}\right) \\
& =2 \delta \varphi_{k} X-\delta\left(\eta_{i}(X) \xi_{j}-\eta_{j}(X) \xi_{i}\right)-\delta \eta_{k}\left(\varphi_{j} X\right) \xi_{j}-\delta \eta_{j}(X) \varphi_{j} \xi_{k} \\
& =2 \delta \varphi_{k} X .
\end{aligned}
$$

Analogously, $\mathcal{L}_{\xi_{j}} \varphi_{i}=-2 \delta \varphi_{k}$.

Theorem 3. Let $\left(M, \varphi_{i}, \xi_{i}, \eta_{i}, g\right)$ be a 3-(0, $\left.\delta\right)$-Sasaki manifold. Then both the horizontal and the vertical distribution are integrable with totally geodesic leaves. Each leaf of the vertical distribution is locally isomorphic to the Lie group $\mathrm{SO}(3)$, with constant sectional curvature $\delta^{2}$; each leaf of the horizontal distribution is endowed with a hyper-Kähler structure. Consequently, the Riemannian Ricci tensor of $M$ is given by

$$
\operatorname{Ric}^{g}=2 \delta^{2} \sum_{i=1}^{3} \eta_{i} \otimes \eta_{i}
$$

Proof. We already know that the horizontal distribution $\mathcal{H}$ is integrable. From (15), for every $X, Y \in \Gamma(\mathcal{H})$ and $i=1,2,3$, we have

$$
g\left(\nabla_{X}^{g} Y, \xi_{i}\right)=-g\left(\nabla_{X}^{g} \xi_{i}, Y\right)=0,
$$

so that the distribution $\mathcal{H}$ has totally geodesic leaves. Furthermore, Equation (16) implies that the vertical distribution $\mathcal{V}$ is also integrable with totally geodesic leaves. In particular $\left[\xi_{i}, \xi_{j}\right]=2 \delta \xi_{k}$ for an even permutation $(i, j, k)$ of $(1,2,3)$, so that the leaves of $\mathcal{V}$ are locally isomorphic to the Lie group $\mathrm{SO}(3)$ and have constant sectional curvature $\delta^{2}$. On each leaf of the horizontal distribution $\mathcal{H}$ one can consider the almost hyper-Hermitian structure defined by $\left(J_{i}:=\left.\varphi_{i}\right|_{H}, g\right)$, which turns out to be hyper-Kähler due to (14). Consequently, $M$ is locally the Riemannnian product of 3-dimensional sphere of curvature $\delta^{2}$ and a $4 n-$ dimensional manifold $M^{\prime}$, which is endowed with a hyper-Kähler structure. Since any hyper-Kähler manifold is Ricci flat, we obtain that the Riemannian Ricci tensor of $M$ is given by (19).

Remark 3. From Theorem 3 it follows that any 3- $(0, \delta)$-Sasaki manifold is locally isometric to the Riemannnian product of 3-dimensional sphere and a 4n-dimensional manifold $M^{\prime}$, which is endowed with a hyper-Kähler structure. We recall that 3- $\delta$-cosymplectic manifolds are also locally isometric to the Riemannian product of a 3-dimensional sphere of constant curvature $\delta^{2}$ and a hyper-Kähler manifold. Nevertheless, there is a difference between the two geometries. Looking at the transverse geometry of the foliation defined by the vertical distribution $\mathcal{V}$, in both cases the Riemannian metric $g$ is projectable, being the vector fields $\xi_{i}, i=1,2,3$, all Killing. In the case of 3- $\delta$-cosymplectic manifolds, each tensor field $\varphi_{i}$ is also projectable, as by (11), the Lie derivatives with respect to the Reeb vector fields satisfy $\left(\mathcal{L}_{\xi_{i}} \varphi_{j}\right) X=0$ for every $i, j=1,2,3$ and for every horizontal vector field $X$. In the case of 3-(0, $\delta)$-Sasaki manifolds, owing to (18), the tensor fields are not projectable. Nevertheless, taking into account the horizontal parts $\Phi_{i}^{\mathcal{H}}:=\Phi_{i}+\eta_{j} \wedge \eta_{k}$ of the fundamental 2-forms $\Phi_{i}$, one can verify that horizontal 4-form

$$
\Phi_{1}^{\mathcal{H}} \wedge \Phi_{1}^{\mathcal{H}}+\Phi_{2}^{\mathcal{H}} \wedge \Phi_{2}^{\mathcal{H}}+\Phi_{3}^{\mathcal{H}} \wedge \Phi_{3}^{\mathcal{H}}
$$

is projectable and defines a transversal quaternionic structure, which turns out to be locally hyper-Kähler. 


\section{Connections with Totally Skew-Symmetric Torsion}

In this section we will show that every 3- $(0, \delta)$-Sasaki manifold is canonical in the sense of the definition given in [1], thus admitting a special metric connection with totally skew-symmetric torsion, called canonical. Recall that a metric connection $\nabla$ with torsion $T$ on a Riemannian manifold $(M, g)$ is said to have totally skew-symmetric torsion, or skew torsion for short, if the $(0,3)$-tensor field $T$ defined by $T(X, Y, Z):=g(T(X, Y), Z)$ is a 3 -form. The relation between $\nabla$ and the Levi-Civita connection $\nabla^{g}$ is then given by

$$
\nabla_{X} Y=\nabla_{X}^{g} Y+\frac{1}{2} T(X, Y)
$$

For more details we refer to [20]. We recall now the definition and the characterization of canonical almost 3-contact metric manifolds.

Definition 4 ([1]). An almost 3-contact metric manifold $\left(M, \varphi_{i}, \xi_{i}, \eta_{i}, g\right)$ is called canonical if the following conditions are satisfied:

(i) each $N_{\varphi_{i}}$ is totally skew-symmetric on $\mathcal{H}$,

(ii) each $\xi_{i}$ is a Killing vector field,

(iii) for any $X, Y, Z \in \Gamma(\mathcal{H})$ and any $i, j=1,2,3$,

$$
N_{\varphi_{i}}(X, Y, Z)-d \Phi_{i}\left(\varphi_{i} X, \varphi_{i} Y, \varphi_{i} Z\right)=N_{\varphi_{j}}(X, Y, Z)-d \Phi_{j}\left(\varphi_{j} X, \varphi_{j} Y, \varphi_{j} Z\right),
$$

(iv) $M$ admits a Reeb Killing function $\beta \in C^{\infty}(M)$, that is the tensor fields $A_{i j}$ defined on $\mathcal{H}$ by

$$
A_{i j}(X, Y):=g\left(\left(\mathcal{L}_{\xi_{j}} \varphi_{i}\right) X, Y\right)+d \eta_{j}\left(X, \varphi_{i} Y\right)+d \eta_{j}\left(\varphi_{i} X, Y\right),
$$

satisfy

$$
A_{i i}(X, Y)=0, \quad A_{i j}(X, Y)=-A_{j i}(X, Y)=\beta \Phi_{k}(X, Y),
$$

for every $X, Y \in \Gamma(\mathcal{H})$ and every even permutation $(i, j, k)$ of $(1,2,3)$.

Here $N_{\varphi_{i}}$ also denotes the (0,3)-tensor field defined by $N_{\varphi_{i}}(X, Y, Z):=g\left(N_{\varphi_{i}}(X, Y), Z\right)$ and we say that $N_{\varphi_{i}}$ is totally skew-symmetric on $\mathcal{H}$ if the $(0,3)$-tensor is a 3 -form on $\mathcal{H}$.

Theorem 4 ([1]). An almost 3-contact metric manifold $\left(M, \varphi_{i}, \xi_{i}, \eta_{i}, g\right)$ is canonical, with Reeb Killing function $\beta$, if and only if it admits a metric connection $\nabla$ with skew torsion such that

$$
\nabla_{X} \varphi_{i}=\beta\left(\eta_{k}(X) \varphi_{j}-\eta_{j}(X) \varphi_{k}\right)
$$

for every vector field $X$ on $M$ and for every even permutation $(i, j, k)$ of $(1,2,3)$. If such a connection $\nabla$ exists, it is unique and its torsion is given by

$$
\begin{aligned}
T(X, Y, Z) & =N_{\varphi_{i}}(X, Y, Z)-d \Phi_{i}\left(\varphi_{i} X, \varphi_{i} Y, \varphi_{i} Z\right), \\
T\left(X, Y, \xi_{i}\right) & =d \eta_{i}(X, Y), \\
T\left(X, \xi_{i}, \xi_{j}\right) & =-g\left(\left[\xi_{i}, \xi_{j}\right], X\right), \\
T\left(\xi_{1}, \xi_{2}, \xi_{3}\right) & =2(\beta-\delta),
\end{aligned}
$$

for every $X, Y, Z \in \Gamma(\mathcal{H})$, and $i, j=1,2,3$, and where $\delta$ is the Reeb commutator function.

The connection $\nabla$ is called the canonical connection of $M$, and also satisfies

$$
\nabla_{X} \xi_{i}=\beta\left(\eta_{k}(X) \xi_{j}-\eta_{j}(X) \xi_{k}\right), \quad \nabla_{X} \eta_{i}=\beta\left(\eta_{k}(X) \eta_{j}-\eta_{j}(X) \eta_{k}\right)
$$

for every vector field $X$ on $M$. Therefore, when $\beta=0$ the canonical connection parallelizes all the structure tensor fields, in which case we call the almost 3-contact metric manifold parallel. 
Both 3- $(\alpha, \delta)$-Sasaki manifolds and 3- $\delta$-cosymplectic manifolds turn out to be canonical. In particular,

Theorem 5 ([1]). Every 3- $(\alpha, \delta)$-Sasaki manifold is a canonical almost 3-contact metric manifold, with constant Reeb Killing function $\beta=2(\delta-2 \alpha)$. The torsion $T$ of the canonical connection $\nabla$ is given by

$$
T=\sum_{i=1}^{3} \eta_{i} \wedge d \eta_{i}+8(\delta-\alpha) \eta_{123}=2 \alpha \sum_{i=1}^{3} \eta_{i} \wedge \Phi_{i}^{\mathcal{H}}+2(\delta-4 \alpha) \eta_{123}
$$

and satisfies $\nabla T=0$.

We denote by $\eta_{123}$ the 3 -form $\eta_{1} \wedge \eta_{2} \wedge \eta_{3}$. From the above theorem, it follows that any 3- $(\alpha, \delta)$-Sasaki manifold is a parallel canonical manifold if and only if $\delta=2 \alpha$, in which case the 3- $(\alpha, \delta)$-Sasaki structure is positive $(\alpha \delta>0)$.

Regarding 3- $\delta$-cosymplectic manifolds, we have:

Proposition 5 ([1]). Any 3- $\delta$-cosymplectic manifold is a parallel canonical almost 3-contact metric manifold. The torsion of the canonical connection is given by

$$
T=-2 \delta \eta_{123}
$$

For the class of 3-(0, $\delta)$-Sasaki manifolds, we have the following

Proposition 6. Every 3-(0, $\delta)$-Sasaki manifold is a canonical almost 3-contact metric manifold, with constant Reeb Killing function $\beta=2 \delta$. The torsion $T$ of the canonical connection $\nabla$ is given by

$$
T=2 \delta \eta_{123}
$$

which satisfies $\nabla T=0$.

Proof. Let $\left(M, \varphi_{i}, \xi_{i}, \eta_{i}, g\right)$ be a 3- $(0, \delta)$-Sasaki manifold. We showed that the structure is hypernormal and the Reeb vector fields are Killing. Furthermore, by the second equation in (12), $d \Phi_{i}(X, Y, Z)=0$ for every $X, Y, Z \in \Gamma(\mathcal{H})$. Therefore, conditions (i), (ii) and (iii) in Definition 4 are easily verified. As regards condition (iv), applying the first equation in (4) and Corollary 1 , for every $X, Y \in \Gamma(\mathcal{H})$ we have

$$
A_{i i}(X, Y)=0, \quad A_{i j}(X, Y)=-A_{j i}(X, Y)=2 \delta \Phi_{k}(X, Y) .
$$

Hence, the structure is canonical with Reeb commutator function $\beta=2 \delta$. Now, by Theorem 4, taking also into account the fact that the vertical distribution is integrable, the only non-vanishing term of the canonical connection is $T\left(\xi_{1}, \xi_{2}, \xi_{3}\right)=2 \delta$, so that $T=2 \delta \eta_{123}$. Although the structure is not parallel when $\delta \neq 0$, the torsion satisfies $\nabla T=0$, as by (20), the 3 -form $\eta_{123}$ is parallel with respect to $\nabla$.

The above result generalizes the result obtained in [2] for the Lie group described in Example 1 (see also Remark 2).

Funding: This research received no external funding.

Conflicts of Interest: The author declares no conflict of interest.

\section{References}

1. Agricola, I.; Dileo, G. Generalizations of 3-Sasakian manifolds and skew torsion. Adv. Geom. 2020, 20, 331-374. [CrossRef]

2. Andrada, A.; Dileo, G. Odd dimensional counterparts of abelian complex and hypercomplex structures. arXiv 2020, arXiv:2006.16435. 
3. Blair, D.E. Riemannian Geometry of Contact and Symplectic Manifolds, 2nd ed.; Progress in Mathematics 203; Birkhäuser: Boston, MA, USA, 2010.

4. Cappelletti-Montano, B.; De Nicola, A.; Yudin, I. A survey on cosymplectic geometry. Rev. Math. Phys. 2013, $25,1343002$. [CrossRef]

5. Boyer, C.; Galicki, C. Sasakian Geometry; Oxford Mathematical Monographs; Oxford Univiversity Press: Oxford, UK, 2008.

6. Yano, K.; Ishihara, S.; Konishi, M. Normality of almost contact 3-structure. Tôhoku Math. J. 1973, 25, 167-175. [CrossRef]

7. Cappelletti-Montano, B.; De Nicola, A.; Yudin, I. Cosymplectic p-spheres. J. Geom. Phys. 2016, 100, 68-79. [CrossRef]

8. Kuo, Y.-Y. On almost contact 3-structure. Tôhoku Math. J. 1970, 22, 325-332. [CrossRef]

9. Boyer, C.P.; Galicki K. 3-Sasakian manifolds. In Surveys in Differential Geometry: Essays on Einstein Manifolds; Wang, M., Lebrun, C., Eds.; International Press: Cambridge, UK, 2007; pp. 123-184.

10. Boyer, C.P.; Galicki, K.; Mann, B.M. The geometry and the topology of 3-Sasakian manifolds. J. Reine Angew. Math. 1994, $455,183-220$.

11. Cappelletti-Montano, B. De Nicola, A. 3-Sasakian manifolds, 3-cosymplectic manifolds and Darboux theorem. J. Geom. Phys. 2007, 57, 2509-2520. [CrossRef]

12. Cappelletti-Montano, B.; De Nicola, A.; Yudin, I. Topology of 3-cosymplectic manifolds. Q. J. Math. 2013, 64, 59-82. [CrossRef]

13. Cappelletti-Montano, B.; De Nicola, A.; Dileo, G. 3-quasi-Sasakian manifolds. Ann. Glob. Anal. Geom. 2008, 33, 397-409. [CrossRef]

14. Cappelletti-Montano, B.; De Nicola, A.; Dileo, G. The geometry of 3-quasi-Sasakian manifolds. Int. J. Math. 2009, 20, 1081-1105. [CrossRef]

15. Kashiwada, T. On a contact 3-structure. Math. Z. 2001, 238, 829-832. [CrossRef]

16. Pastore, A.M.; Falcitelli, M.; Ianus, S. Riemannian Submersions and Related Topics; World Scientific Publishing Co., Inc.: River Edge, NJ, USA, 2004.

17. Agricola, I.; Dileo, G.; Stecker, L. Homogeneous non-degenerate 3- $(\alpha, \delta)$-Sasaki manifolds and submersions over quaternionic Kähler spaces. arXiv 2020, arXiv:2011.13434.

18. Konishi, M. On manifolds with Sasakian 3-structure over quaternion Kaehler manifolds. Kodai Math. Semin. Rep. 1975, 26, 194-200. [CrossRef]

19. Tanno, S. Remarks on a triple of K-contact structures. Tôhoku Math. J. II. Ser. 1996, 48, 519-531. [CrossRef]

20. Agricola, I. The Srní lectures on non-integrable geometries with torsion. Arch. Math. (Brno) 2006, 42, 5-84. 